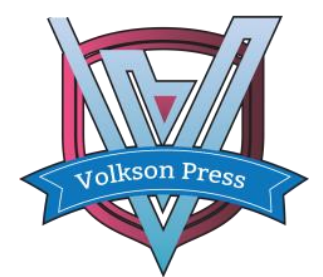

Contents List available at VOLKSON PRESS

Clean Water,Air\&Soil(CleanWAS)

DOI : http://doi.org/10.26480/cleanwas.01.2017.27.29

\title{
Multi-parameter custom critical defect evaluation method of Ultrasonic Testing Method
}

Liu $\operatorname{Lin}^{a b^{*}}$

\author{
${ }^{a}$ College of Resource and Civil Engineering, Northeastern University, Shenyang, 110000, China, bivil Engineering College, Shenyang jianzhu \\ University, Shenyang, 110000, China, \\ *Corresponding Author: Liu Lin, E-mail: liumumu118@163.com
}

This is an open access article distributed under the Creative Commons Attribution License, which permits unrestricted use, distribution, and reproduction in any medium, provided the original work is properly cited

\section{ARTICLE DETAILS}

\section{Article History:}

Received 02 october 2017 Accepted 06 october 2017 Available online 11 october 2017

\section{Keywords:}

Multi-parameter, custom defect discrimination threshold, Ultrasonic Testing

\section{ABSTRACT}

For several situations encountered in the detection, more complex internal structure, or testing data of smaller discreteness, data is less or more close, which is difficult to meet the conditions of use, that is concrete defect discrimination method of the ultrasonic testing method of current Chinese specifications. This paper aimed at existing in the detection of the above situation, studied the problems of the existing specifications defect discrimination method in solving the above situation, studied a multi-parameter custom critical defect evaluation method, respectively, studied on the use of the method in the testing data of smaller discreteness and complex internal structure. As detection is shown, first, this method can improve the accuracy in ultrasonic testing in such case, can effectively avoid the false negatives. IT makes defect detection rate of $90 \%$ or more. Second, according to the waveform of abnormalities detection data to determine defect discrimination threshold, it determines the defects of parts and the final defect result is more close. In addition, For conditions with less testing data, the use of other testing means, compares the ultrasonic testing results to obtain acoustic parameters, in order to set custom defect discrimination threshold, and reduce the number of false negatives.

\section{Introduction}

Sometimes, due to the leakage of vibration or rocks overhead on the steel skeleton, during construction, there is some concrete honeycomb or hollow in reinforced concrete structures. Especially for the concrete of the thicker reinforced parts, such as the frame structure of beam and column node, it is more complex for the construction. The difficulties would be encountered in aspects of the steel bar arrangement and concrete pouring. Because of reinforced dense, there is certain influence on the liquidity of concrete, and the concrete material flow are cut off, the lower part of the concrete has not been vibrating, the upper concrete is continue to cast. Those are very well lead to loose region, cave, light aggregate and coarse aggregate be uneven exist in concrete structure. These defects influence seriously loading ability and durability of concrete structure. The quality control of concrete, in addition to the optimization of the mix proportioning of the concrete, choose the reasonable way of casting and strict controls of construction technology, etc, another important way is to strengthen the physical quality inspection. Using nondestructive testing technology, timely find the lesions during the construction of the built or building, through the fast repair technology to reinforce of defective parts, to avoid catastrophic accidents, which undoubtedly has the good social efficiency and economic benefits.

At present, the ultrasonic testing method as a nondestructive detecting method is widely applied to concrete defect detection. Compared with other NDT methods, ultrasonic testing reveals the advantages which have the ability of penetrating power. Ultrasonic nondestructive testing method not only for the structure itself does not cause damage, but also economical and practical. But the existing defect discrimination method of the ultrasonic testing method of current Chinese specifications is not very applicable, for several situations encountered in the detection, more complex internal structure, or testing data of smaller discreteness, data is less or more close. It is difficult to meet the conditions of use, easy to cause the defect missing. In view of the using problems of the existing ultrasonic defect discrimination method, put forward a method based on probability method, which is the multi-parameter custom critical defect evaluation method.

\section{Ultrasonic inspection principle}

When raw material, mix design, quality and testing distance of concret is same, propagation velocity, magnitude, frequency of ultrasonic wave should be concerned properly. By measuring in concrete ultrasonic pulse velocity, the first wave of received signal amplitude and frequency acoustic parameters, and so on. According to these parameters and the relative changes, judge the defects of concrete (the concrete inner cavity and imperfect of the location and scope, crack depth, surface damage different thickness, time of concrete pouring junction plane quality, the pile and concrete filled steel tube defects such as).

In 2000, China promulgated the newly revised "TECHNICAL SPECIFICATION FOR INSPECTION OF CONCRETE DEFECTS BY ULTRASONIC METHOD" [CECS 21:2000]. The probability method (Chinese Standards 2010) is proposed. It get into a arrangement of propagation velocity ,magnitude, frequency which are all testing point in the surveyed according to number, then, in the arrangement, the doubtful value is the smallest data that can be referred to, then, including the largest doubtful value and all normal dates, average $\mathrm{mx}$ and root-mean-square value sx was calculated, then, the abnormal judgment value $\mathrm{X} 0$ which is adopted to test defect value is calculateded by Eq. 1:

$$
\chi_{0}=m_{x}-\lambda_{1} \cdot s_{x}
$$

Where $m$ is average value, $s_{x}$ is root-mean-square value. Then, comparing the abnormity value $\chi_{0}$ with the largest doubtful value $\chi_{n}$, when $\chi_{n}$ is less than or equal to $\chi_{0}, \chi_{n}$ and all arranged in $\chi_{n}$ subsequent data are abnormal value. When $\chi_{n}$ is more than $\chi_{0}$, we should start a new set of statistical computation and discrimination with $\chi_{n+1}$ and all normal dates. Finally, when some propagation velocity, magnitude, frequency are diagnosed as abnormal, the concrete inner cavity and imperfect of the location and scope can be approximately determined by using abnormity status distribution and wave forms. 
normal distribution. Because of gross error, it is not follow normal distributions.

\section{Problems}

There are certain requirements in the specification for the use of probability method. Including the same condition (component, age, concrete proportioning and materials, testing ranging conditions) of ultrasonic data should not be less than 10 , defect data points are not to participate in the calculation $m_{x}$ and $s_{x}$. So ultrasonic defect judgment method of specification, which is not fit to the defect area is larger in detect area, or less effective data available. Those made overall quality of members be poor, the discreteness of data be less, causes low judgment value, and the defects to leakage. In addition, the less data is not easy to found out the outliers. At the same time, the complex environment, the same condition normal concrete point is difficult to meet the requirements of quantity.

\section{The multi-parameter custom critical defect evaluation method}

We put forward a method based on probability method, which is the multiparameter custom critical defect evaluation method. The judging method of internal defects of concrete structure is divided into two steps. The first step, we use the probabilistic method to judge the defect position preliminary, and using the drilling or radar method judging whether there is missing information. The second step, when there are leakage of common defects, need to set the parameters defined critical value, supplementary judgment. This can reduce the missing, improve efficiency.

\subsection{Multi-parameter custom threshold setting method}

First of all, respectively, to achieve the following three conditions multiparameter custom threshold.

Through ultrasonic testing for normal concrete in the same condition, obtain sound velocity, amplitude, frequency be calculated by Eq. (1) to determine the sound velocity, amplitude or frequency of multi-parameter custom is $\chi_{a}$.

According to the data of waveform, combined with the waveform abnormalities, to get the maximum value of abnormal waveform data, and to set multi parameter custom defect discrimination value is $\chi_{b}$.

Compared with other methods, such as the core method of core samples. Obtained acoustic parameters of core samples, or near the core sample did not destroy the position of the acoustic parameters, then be calculated by Eq. (1) to determine the sound velocity, amplitude or frequency of multiparameter custom is $\chi_{c}$.

Then, the above three conditions multi-parameter custom thresholds compare the abnormity value $\chi_{0}$ that shall be calculated by Eq. (1) in the first step. The maximum value of each acoustic parameters, were set to multi-parameter custom threshold, $\chi$ shall be calculated by Eq. (2).

$\chi=\chi_{\max }\left(\chi_{a}, \chi_{b}, \chi_{c}, \chi_{0}\right)$

Finally, combined with the abnormal data waveform abnormalities, waveform anomalies can judge here for the defect.

\subsection{The application of multi-parameter custom critical defect evaluation method in data with low dispersion}

We adopted ultrasonic angle measurement method for concrete jointing surface of one pillar testing analysis. The new pouring concrete sound velocity is less than below the surface. The combining surface is for the majority of poor bond. The new pouring concrete aggregate accumulation is in the old concrete surface. The partial position concrete couldn't take the full core samples. We used multi-parameter custom critical defect evaluation method for the combination of surface defect judgment.

First of all, the method of probability analysis was carried out on the data collected. The abnormal judgment value $\chi_{0}$ was calculated by Eq. (1), including the sound velocity, amplitude or frequency of multi-parameter custom in Table 1. Defective part is not obvious. There are 9 abnormal data, see figure 1.

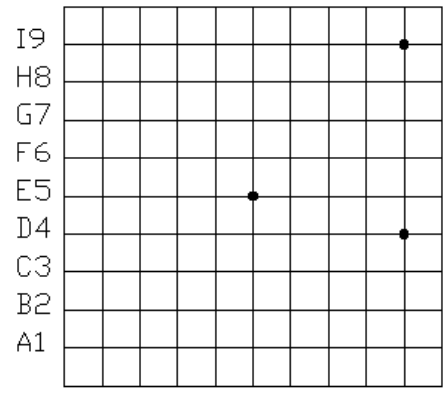

1234456789

Figure1. Specification probability method judging defect

Secondly, through ultrasonic testing for normal concrete in the same condition, obtain sound velocity, amplitude, frequency be calculated by Eq.

(1) to determine the sound velocity, amplitude or frequency of multiparameter custom was $\chi_{a}$ in Table 1.

Thirdly, according to the data of waveform, combined with the waveform abnormalities, to get the maximum value of abnormal waveform data, and to set multi parameter custom defect discrimination value was $\chi_{b}$ in Table

Table 1. Four cases of multi-parameter custom threshold

\begin{tabular}{cccc}
\hline $\begin{array}{c}\text { The abnormal } \\
\text { judgment value }\end{array}$ & $\begin{array}{c}\text { Velocity value } \\
\mathrm{Km} / \mathrm{s}\end{array}$ & $\begin{array}{c}\text { amplitude } \\
\text { value dB }\end{array}$ & $\begin{array}{c}\text { frequency } \\
\text { value } \\
\mathrm{KHz}\end{array}$ \\
\hline$\chi_{0}$ & 3.892 & 38.4 & 37.4 \\
$\chi_{a}$ & 4.3 & 48.3 & 44.5 \\
$\chi_{b}$ & 4.6 & 46 & 43 \\
$\chi_{c}$ & 3.12 & 42.5 & 39.3 \\
\hline
\end{tabular}

Fourthly, compared with core samples, obtained acoustic parameters of core samples and near the core sample that wasn't destroyed, then be calculated by Eq. (1) to determine the sound velocity, amplitude or

frequency of multi-parameter custom is $\chi_{b}$ in Table 1.

Fifthly, the maximum value of each acoustic parameters, were set to multiparameter custom threshold, $\chi$ shall be calculated by Eq. (2) . Finally, combined with the data waveform, and ultimately determine the defects as shown in figure 2, arrow is for drilling for core samples. Core sample is shown in figure 3 , we can clearly see the core samples with joint.

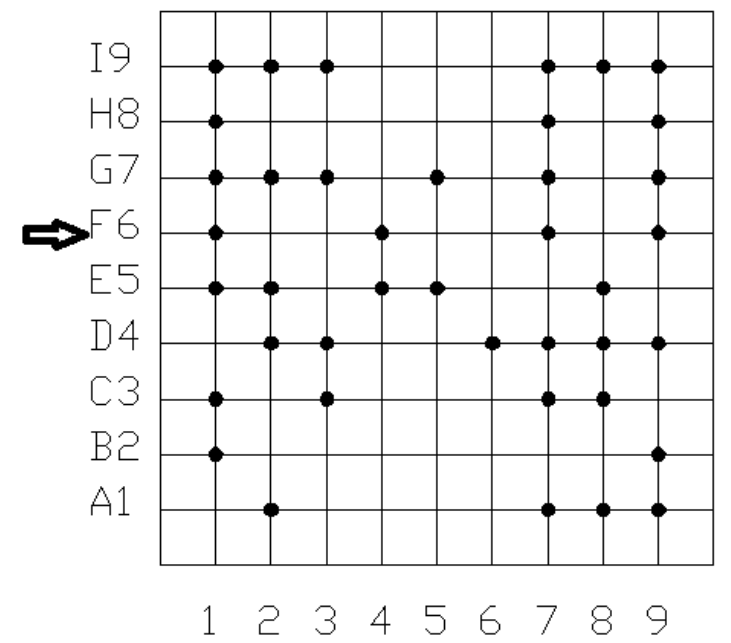

Figure 2.The application of multi-parameter custom critical defect evaluation

method judging defect 


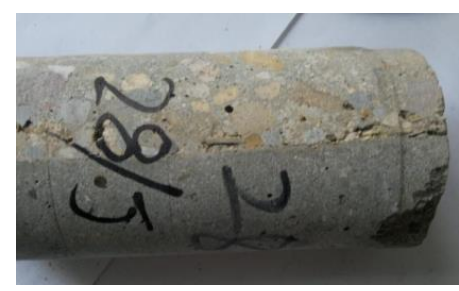

Figure 3. The sample of the drilling core

Due to the use or the acoustic parameters or normal concrete, the custom critical value will be higher. It can cause excess of judgment, comparing the actual defect results detection. So, it is very important for the recognition of waveform data. In addition, by comparing a large amount of data, we found that using $\chi_{b}$ to directly define $\mathrm{X}$, its final defect result is more close the actual. But considering the difference discrimination waveform, we suggest using the defects of improved discrimination method in this paper to define $\chi$.

\subsection{The application of multi-parameter custom critical defect evaluation method in the same conditions less data}

The building is located in Shenyang China. The node of steel reinforced concrete column and steel beam structure were detected. There were using ultrasonic, radar method and drilling method for comprehensive detection. The different test results were analyzed.

Normal node. The waveform is shown in figure 4 . The velocity of sound is in the above $4.2 \mathrm{~km} / \mathrm{s}$. Radar chart is shown in figure 5 , for the normal parts ( Liu et al 2013).

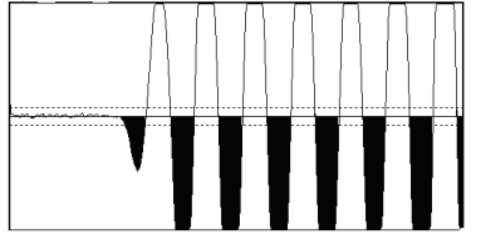

Figure 4. Normal parts ultrasonic wave

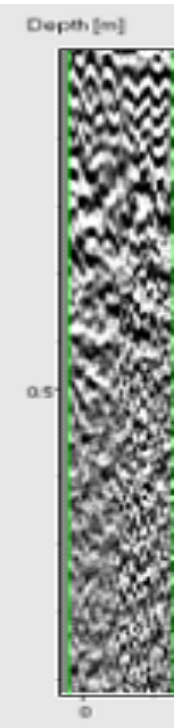

Figure 5. Normal parts radar chart

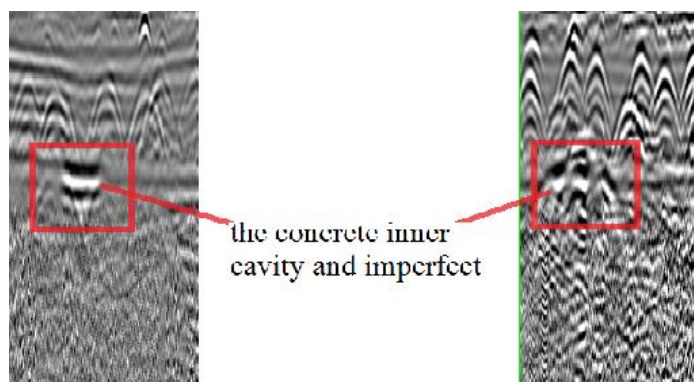

Figure 7. cavity and imperfect radar chart

Steel and concrete separately. The waveform is shown in figure 8. The velocity of sound is between $2.5-4.2 \mathrm{~km} / \mathrm{s}$. The first wave was very difficult to obtain in the position of cavity. Abnormal parts were by drilling and sampling. The gap between the concrete and steel is very small.

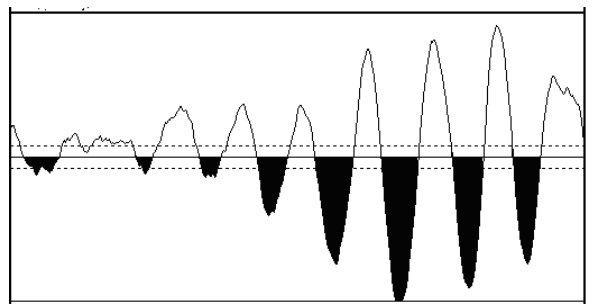

Figure 8. Steel and concrete separately ultrasonic wave

According to test and analysis result, the same condition normal concrete point is difficult to meet the requirements of quantity, which is not fit to ultrasonic defect judgment method of specification. In addition, when a defect is detected, velocity will decrease. The velocity of sound is in $4.2 \mathrm{~km} / \mathrm{s}$. It can be seen from the data. Velocity is less than $3.5 \mathrm{~km} / \mathrm{s}$, which part is cavity and imperfect, or steel and concrete separately. IT is very difficult to distinguish type of defect, and which reinforcement measures are to take. But when we analysis ultrasonic waveform, according to whether the first wave can be obtained, the types of defects can be distinguished. So, we used multi-parameter custom critical defect evaluation method, set multi parameter custom defect discrimination value was $\chi_{b}$, and analysis ultrasonic waveform.

The discrimination effects are very significant, on big proportion of the disease, or detect data similar. IT makes defect detection rate of $90 \%$ or more.

By comparing a large amount of data, we found that using $\chi_{b}$ to directly define $\mathrm{X}$, its final defect result is more close the actual. But considering the difference discrimination waveform, we suggest using the defects of improved discrimination method in this paper to define $\chi$

For the less detection data of the same conditions in structure joints, acoustic parameters can be obtained by other methods comparison of ultrasonic detection, which is set custom defect discrimination threshold.

Cavity and imperfect. The waveform is shown in figure 6 . The velocity of 5 . sound is in $4.2 \mathrm{~km} / \mathrm{s}$. The first wave was very difficult to obtain in the $\mathbf{6}$. position of cavity. Radar chart is shown in figure 7.

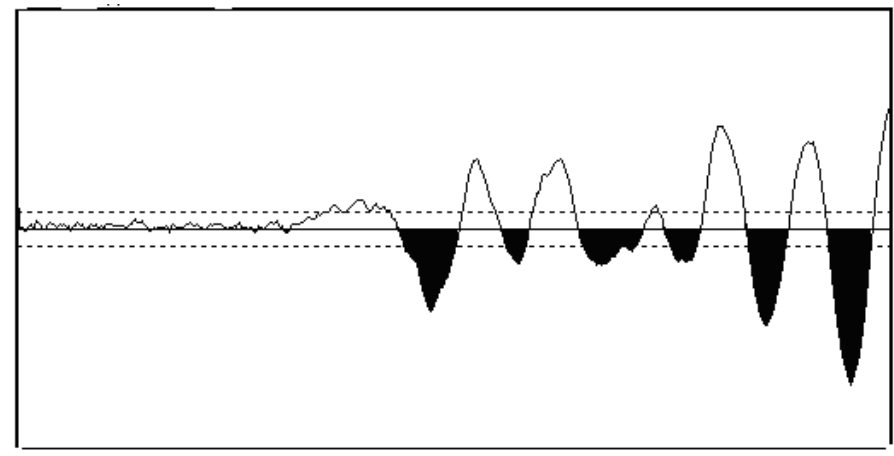

Figure 6. cavity and imperfect ultrasonic wave

\section{Conclusions}

This paper introduces an improved defect evaluation method, which is in view of the using ultrasonic defect discrimination method, and put forward the multi-parameter custom critical defect evaluation method. As with the new method is used, several situations encountered in the detection, that is more complex internal structure, or testing data of smaller discreteness, defect discrimination effect is very significant.

\section{References}

China Engineering Construction Standardization Association standard. (2000). technological specification for inspection of concrete defects by ultrasonic method, [CECS 21:2000], BeiJin.

Liu, L., \& Zhao, W.(2013), Large Ranging of Ultrasonic Testing Inspection Internal Defects of Mass Concrete Structure. Applied Mechanics and Materials, 239,1492-1495.

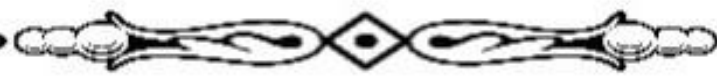

